UDC 621.039.674.3

\title{
SUBCRITICALITY MONITORING IN FUSION-FISSION HYBRID REACTORS
}

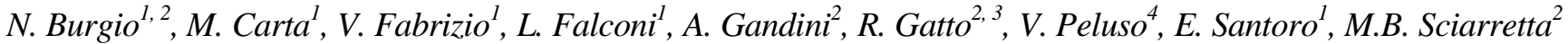 \\ ${ }^{I}$ ENEA CRE Casaccia, Santa Maria di Galeria, Italy \\ ${ }^{2}$ Sapienza University of Rome, DIAEE, Corso Vittorio Emanuele, Roma, Italy \\ ${ }^{3}$ CR Enrico Fermi, Roma, Italy \\ ${ }^{4}$ ENEA CR Bologna, Bologna, Italy
}

Based on the heuristically generalized perturbation theory (HGPT) adapted to subcritical systems [1], a procedure for online operational monitoring of the subcriticality level of a hybrid fusion-fission system in which an "external" neutron source is generated in a magnetically confined tokamak-type plasma is described. This procedure [2], commonly referred to as power control-based subcriticality monitoring (PCSM), consists of compensating of slow and small movements of a specialized control rod in the nuclear fission zone, previously calibrated using a standard procedure, with equally slow and small alterations of the fusion neutron source. PCSM is verified by solving the multigroup transport equation for the direct flux, as well as for the importance function, associated with normalized fission power [3], and a technique is proposed for modifying the fusion neutron source strength based on plasma compression/expansion. Plasma and confining magnetic field adjustments, needed to implement the PCSM, are estimated with due account of the 0-D-plasma power balance.

Key words: fusion-fission hybrid reactor, subcritical fission core, subcritical multiplication factor, reactivity monitoring.

DOI: 10.21517/0202-3822-2021-44-2-27-41

\section{КОНТРОЛЬ ПОДКРИТИЧНОСТИ В ГИБРИДНЫХ РЕАКТОРАХ СИНТЕЗА-ДЕЛЕНИЯ}

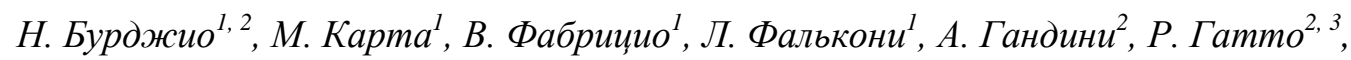 \\ В. Пелузо ${ }^{4}$, Е. Санторо ${ }^{1}$, М.Б. Шьяретта ${ }^{2}$
}

\begin{abstract}
${ }^{1}$ Национальное агентство по новым технологиям, энергии и устойчивому экономическому развитию (ENEA), Исследовательский центр (ИЦ) Касаччиа, ул. Ангильярезе 301, 00123 Санта-Мария-ди-Галерия, Италия

${ }^{2}$ Сапиенияа - Римский университет, Отделение космической электротехники и энергетики, проспект Виктора-Эммануила II 244, 00186 Рим, Италия

${ }^{3}$ ИЦ им. Энрико Ферми, ул. Панисперна 89а, 00184 Рим, Италия

${ }^{4}$ ENEA, Болонский ИЦ, ул. Мартири Монте Соле 4, 40129 Болонья, Италия
\end{abstract}

На основе распространения методологии HGPT (Эвристически обобщённой теории возмущений) на подкритические системы [1] описана процедура оперативного мониторинга уровня подкритичности гибридной системы синтеза-деления, в которой внешний источник нейтронов генерируется в магнитно-замкнутой плазме типа токамак. Процедура [2], обычно называемая методом PCSM (Power Control-Based Subcritality Monitoring), заключается в компенсации медленных малых перемещений специализированного регулирующего стержня в зоне деления ядер, предварительно откалиброванного с использованием стандартной процедуры, такими же медленными малыми изменениями источника термоядерных нейтронов. Метод верифицируется путём решения многогруппового уравнения переноса как для прямого потока, так и для функции важности. Последняя связана с нормированной мощностью деления [3]. Предлагается процедура изменения силы источника термоядерных нейтронов на основе сжатия/расширения плазмы. C учётом 0-D-баланса мощности плазмы дана оценка изменения параметров плазмы и ограничивающего магнитного поля, необходимого для реализации метода PCSM.

Ключевые слова: гибридный реактор синтеза-деления, подкритическая зона деления ядер, подкритический коэффициент размножения, измерение реактивности.

\section{INTRODUCTION}

A fusion-fission hybrid system consists in a fusion part, where fusion nuclear reactions taking place in a hightemperature plasma generate neutrons, and a surrounding subcritical fission core. When fusion power is based on $\mathrm{D}$-T-reactions, as assumed in the present work, an additional tritium production zone is required. The fission core, where neutrons can induce fission events or undergo radiative capture reactions, offers two main potential applications and benefits that could be pursued: radioactive waste management, and nuclear fuel breeding. Thanks to their high energy, fusion-generated neutrons are particularly suitable for coupling with a fast-spectrum fission core to create an optimal environment for fertilization and nuclear waste burning. Fission nuclear energy is also produced, even though this is not nowadays regarded as the hybrid system's priority. As for waste management aspect, the most interesting thing is the possibility to achieve the so-called «deep-burn» mode of operation. The «external» fusion neutron source, independent with respect to the fission core, can be used to substantially increase 
the fuel burnup compared to a critical reactor, by maintaining the neutron chain reaction longer, for comparable enrichment. In principle, with an extremely deep burnup it may be possible to employ a once-through «burn and bury» fuel cycle that would eliminate, or drastically reduce, the need for reprocessing and enrichment [4].

Due to its potential promise in addressing the issue of the long-term sustainability of nuclear energy, the fission-fision combination, initially conceptualized in the 1950's [5-7], has been periodically revisited, and today keeps being the subject of theoretical studies and experimental exploration. Several fusion-fission hybrid concepts have been proposed, differing in the plasma confinement scheme and the fission core material [8]. In this work, we focus on magnetically confined plasmas, particularly the high-temperature deuterium-tritium (D-T)-plasma confined in a tokamak device by a combination of a toroidal and a poloidal magnetic field (with the latter selfgenerated by plasma current). An externally generated vertical magnetic field is required to keep the plasma in equilibrium and to conveniently shape it for improved performance. While the prospect of implementing a pure fusion power plant based on the tokamak concept remains distant, a hybrid system, for which the physical and engineering requirements are much less stringent, can be seen as an intermediate step in the quest for fusion energy, plus a way to drive fission energy toward a more sustainable future.

Any subcritical system requires a dedicated control system, capable of a real-time monitoring of the fission core subcriticality. This study is concerned with the feasibility of applying the so-called «power control-based subcriticality monitoring (PCSM)», already discussed in the context of ADS systems [2,3], to fusion-fission hybrids based on the tokamak concept. Formally, the theoretical arguments undrelying the proposed monitoring procedure apply equally to ADS and fusion driven systems. The hybrid's novelty is in the interplay between fission and fusion powers. We propose a method for adjusting fusion power to values consistent with the PCSM procedure using plasma compression/expansion through small alterations of the confining magnetic field.

This paper is formatted into two main parts. In the first part, we carry out a numerical verification of the PCSM method by neutronic calculations done with the ERANOS code [9]. For a credible evaluation of the PCSM effectiveness in monitoring a nuclear system subcriticality, we used a fairly accurate model of the core of a real-life research reactor, viz., TRIGA-RC1, run at the ENEA-Casaccia Research Center [10]. The JEFF-3.1 nuclear data library, adopted in the lattice cell calculations for collapsing the fine energy group cross-section structure into one of 33 groups, was used for Sn neutron transport calculations (BISTRO code [11]) of the direct flux and the importance function. This was the operating environment for the PCSM execution and subcritical reactivity measurement. In the second part of this work, we describe the method for modulating the strength of the fusion neutron source, required to implement the PCSM procedure. To this end, we set up a simple model of a fusion-fission hybrid, based on a 1D cylindrical geometry representation. The internal cavity filled with plasma is overlaid with five zones. They are, starting from the one closest to the plasma, the first wall, the fission core, the fusion blanket, the reflector and the shield. The neutronic calculations are repeated in this simpler setting, and the method to vary the fusion neutron source is implemented. The latter consists in compressing/expanding the plasma by changing the strength of the confining magnetic field.

Following this Introduction, the paper presents a brief overview of the PCSM method and the expression for the subcritical multiplication factor. The next section is concerned with neutronic calculations based on the TRIGA-RC1 reactor modelization. The reactor's layout and main characteristics are provided, the reference and perturbed fission core configurations are defined, and the multiplication factor is calculated according to the PCSM formula. In the section that follows we describe two plasma compression methods for modulating the fusion neutron source, as required by the PCSM procedure. After describing a simplified hybrid system, we define the plasma operating regime with due account of the plasma power balance, and determine plasma's key characteristics, including the strength of the fusion neutron source. Two plasma compression methods are proposed: number one, described in detail in the respective subsection of the «Fusion Neutron Source Modulation» section, relies on the plasma inward displacement. Number two, described only succinctly in the same section, consists in the direct reduction of the plasma minor radius, with the center point kept fixed. The last section is titled «Summary and Conclusions».

\section{SUBCRITICAL SYSTEMS AND THE PCSM METHOD}

The PCSM is rooted in the point kinetics equation related to subcritical systems [1,2].

Point kinetics equation for a subcritical system. The point kinetics equations related to a subcritical system are derived from equations governing neutron flux vector $\Phi$ and precursor densities $m_{i}(i=1,2, \ldots, M)$, with $M$ representing the number of delayed neutron groups, each characterized by decay constant $\lambda_{i}$ under transient conditions in a multi-group neutron energy setting (the bold-face characters are for vectors and matrices): 


$$
\begin{gathered}
\mathbf{V}^{-1} \frac{\partial \boldsymbol{\Phi}}{\partial t}=\mathbf{A} \boldsymbol{\Phi}+(1-\beta) \chi^{\mathrm{P}} \mathbf{S}_{\mathrm{fis}} \mathbf{\Phi}+\chi^{\mathrm{D}} \mathbf{U} \sum_{i=1}^{M} \lambda_{i} m_{i}+\mathbf{S} \\
\frac{\partial m_{i}}{\partial t}=\beta_{i}\left(v \boldsymbol{\Sigma}_{f}\right)^{\mathrm{T}} \mathbf{\Phi}-\lambda_{i} m_{i} .
\end{gathered}
$$

Here $\mathbf{A}$ is the transport (diffusion), capture and scattering matrix operator (of a $G \times G$ dimension, where $G$ denotes the number of energy groups), $\mathbf{V}$ is the diagonal neutron velocity matrix $(G \times G)$, and $\mathbf{U}$ is the unit $(G$ component) vector: $\mathbf{S}_{\mathrm{fis}}=\left[\begin{array}{lll}\left(v \Sigma_{f}\right)_{1} & \mathrm{~L} & \left(v \Sigma_{f}\right)_{G} \\ \mathrm{~L} & \mathrm{~L} & \mathrm{~L} \\ \left(v \Sigma_{f}\right)_{1} & \mathrm{~L} & \left(v \Sigma_{f}\right)_{G}\end{array}\right]_{G \text { rows }}$ is the fission matrix, $\left(v \Sigma_{f}\right)^{T}=v\left[\begin{array}{lll}\Sigma_{f, 1} & \mathrm{~L} & \Sigma_{f, G}\end{array}\right]$, and the prompt $(P)$ and delayed $(D)$ spectra are $\chi^{X}=\operatorname{diag}\left[\begin{array}{lll}\chi_{1}^{X} & \mathrm{~L} & \chi_{G}^{X}\end{array}\right]$ with $\chi=P$ or $D$.

We assume that the transient is initiated at a given time by a perturbation that generally changes unperturbed operators $\mathbf{A}_{0}, \mathbf{S}_{\mathrm{fis}, 0}$, and neutron source $\mathbf{S}_{0}$ into $\mathbf{A}\left(=\mathbf{A}_{0}+\delta \mathbf{A}\right), \mathbf{S}_{\mathrm{fis}}\left(=\mathbf{S}_{\mathrm{fis}, 0}+\delta \mathbf{S}_{\mathrm{fis}}\right), \mathbf{S}\left(=\mathbf{S}_{0}+\delta \mathbf{S}\right)$, respectively, and transforms unperturbed neutron flux $\boldsymbol{\Phi}_{0}$ into $\boldsymbol{\Phi}\left(=\boldsymbol{\Phi}_{0}+\delta \Phi\right)$.

To arrive at an expression accommodating the generalized reactivity derived in the context of the HGPT methodology, we introduce importance function $\Psi^{*}$, associated with normalized fission power, which is governed by equation $\mathbf{A}_{0}^{*} \boldsymbol{\Psi}^{*}+\mathbf{S}_{\mathrm{fis}, 0}^{\mathrm{T}}\left[(1-\beta) \chi^{\mathrm{P}}+\beta \chi^{\mathrm{D}}\right] \boldsymbol{\Psi}^{*}+\frac{\gamma}{W_{0}} \boldsymbol{\Sigma}_{f, 0}=0$. The importance relative to the precursor densities is conveniently defined as $m^{*}=\mathbf{U}^{\mathrm{T}} \boldsymbol{\chi}^{\mathrm{D}} \Psi^{*}$.

After multiplying Eqs. (1) and (2) on the left by $\Psi^{* \mathrm{~T}}$ and $m^{*}$, respectively, space-integrating on the fission core volume, dropping second order terms, and doing some algebra, we obtain (assuming that the neutron flux showing up in the ratios (numerator and denominator) is unperturbed, and accounting for the general dependence of the delayed neutron distribution on nuclide species and energy [1])

$$
\begin{gathered}
1_{\text {eff }} \frac{d P}{d t}=\left(\rho_{\text {gen }}-\alpha \beta_{\mathrm{eff}}\right) P+\sum_{i=1}^{M} \lambda_{i} \xi_{i}+\zeta(1-P)+\rho_{\text {source }} ; \\
\frac{d \xi_{i}}{d t}=\alpha \beta_{\mathrm{eff}, i} P-\lambda_{i} \xi_{i},
\end{gathered}
$$

where the coefficients represent physically significant quantities given by expressions: $P(t)=\frac{W(t)}{W_{0}(1+q)}$, with $q=\frac{\left\langle\delta \boldsymbol{\Sigma}_{f}^{\mathrm{T}}, \boldsymbol{\Phi}_{0}\right\rangle}{\left\langle\boldsymbol{\Sigma}_{f, 0}^{\mathrm{T}}, \boldsymbol{\Phi}_{0}\right\rangle}$ (normalized power); $\xi_{i}=\frac{\left\langle m^{*}, m_{i}\right\rangle}{\left\langle\boldsymbol{\Psi}^{*}, \hat{\boldsymbol{\chi}} \mathbf{S}_{\mathrm{fis}, 0} \boldsymbol{\Phi}_{0}\right\rangle} \quad(i$-th effective precursor density); $1_{\text {eff }}=\frac{\left\langle\Psi^{*}, \mathbf{V}^{-1} \mathbf{\Phi}_{0}\right\rangle}{\left\langle\Psi^{*}, \hat{\chi} \mathbf{S}_{\text {fis, } 0} \boldsymbol{\Phi}_{0}\right\rangle}$ (effective prompt neutron lifetime), where $\hat{\chi} \mathbf{S}_{\text {fis }, 0} \boldsymbol{\Phi}_{0}$ is the unperturbed fission source:

$$
\rho_{\mathrm{gen}}=\frac{\left\langle\boldsymbol{\Psi}^{*},\left(\delta \mathbf{A}+\hat{\chi} \delta \mathbf{S}_{\mathrm{fis}}\right) \boldsymbol{\Phi}_{0}\right\rangle}{\left\langle\boldsymbol{\Psi}^{*}, \hat{\chi} \mathbf{S}_{\mathrm{fis}, 0} \boldsymbol{\Phi}_{0}\right\rangle} \quad \text { (generalized reactivity) }
$$

$\rho_{\text {source }}=\frac{\left\langle\boldsymbol{\Psi}^{*}, \delta \mathbf{S}_{n}\right\rangle}{\left\langle\Psi^{*}, \hat{\chi} \mathbf{S}_{\mathrm{fis}, 0} \boldsymbol{\Phi}_{0}\right\rangle}$ (source reactivity), $\alpha=\frac{\left\langle\boldsymbol{\Psi}^{*}, \chi^{\mathrm{D}} \mathbf{S}_{\mathrm{fis}, 0} \boldsymbol{\Phi}_{0}\right\rangle}{\left\langle\boldsymbol{\Psi}^{*}, \hat{\chi} \mathbf{S}_{\mathrm{fi}, 0} \boldsymbol{\Phi}_{0}\right\rangle}, \zeta=\frac{1}{\left\langle\boldsymbol{\Psi}^{*}, \hat{\chi} \mathbf{S}_{\mathrm{fis}, 0} \boldsymbol{\Phi}_{0}\right\rangle} \equiv \frac{1-k_{\mathrm{sub}}}{k_{\mathrm{sub}}}$, and $\beta_{i, \text { eff }}=\frac{\sum_{j=1}^{\mathrm{J}} \beta_{i}^{j}\left\langle\boldsymbol{\Psi}^{*}, \boldsymbol{\chi}_{i}^{\mathrm{D}, j} \mathbf{S}_{\mathrm{fis}, 0} \boldsymbol{\Phi}_{0}\right\rangle}{\left\langle\boldsymbol{\Psi}^{*}, \hat{\chi} \mathbf{S}_{\mathrm{fis}, 0} \boldsymbol{\Phi}_{0}\right\rangle}, \quad \beta_{\mathrm{eff}}=\sum_{i=1}^{\mathrm{M}} \beta_{i, \text { eff }}$ for $J$ fissile isotopes. The subcritical multiplication factor $k_{\mathrm{sub}}=\frac{\left\langle\boldsymbol{\Psi}^{*}, \hat{\chi} \mathbf{S}_{\mathrm{fis}, 0} \boldsymbol{\Phi}_{0}\right\rangle}{1+\left\langle\boldsymbol{\Psi}^{*}, \hat{\chi} \mathbf{S}_{\mathrm{fis}, 0} \mathbf{\Phi}_{0}\right\rangle}$. 
In the above expressions, $\gamma$ is energy released in one fission event, and $\hat{\chi}=\chi^{\mathrm{P}}(1-\beta)+\chi^{\mathrm{D}} \beta$ is the neutron fission spectrum consisting of the prompt and the delayed components ( $\beta$ is the delayed neutron fraction). It can be shown that $k_{\text {sub }}$ merges into $k_{\text {eff }}$ (the multiplication factor related to the fundamental eigenfunction) as the system approaches criticality [1].

Eqs. (3) and (4) can be considered as a generalization of the point kinetics system equation derived by Usachev for critical systems [12], that converge with the Usachev's equation when the system approaches criticality.

The PCSM method. Assume that a dedicated control rod has been calibrated so that its displacement $\Delta z$ may be associated with (experimental) reactivity values $\left(\Delta k_{\mathrm{eff}} / k_{\mathrm{eff}}\right)^{\mathrm{exp}}$. The associated $\rho_{\mathrm{gen}}^{\mathrm{exp}}$ of the (experimental) generalized reactivity can be assumed to be given as follows:

$$
\rho_{\mathrm{gen}}^{\mathrm{exp}}=\rho_{\mathrm{gen}}^{\mathrm{calc}} f_{\mathrm{b}},
$$

with $\rho_{\text {gen }}^{\text {calc }}$ given by Eq. (5), and the bias factor, $f_{\mathrm{b}}=\frac{\left(\Delta k_{\mathrm{eff}} / k_{\mathrm{eff}}\right)_{\mathrm{B}}^{\text {exp }}}{\left(\Delta k_{\mathrm{eff}} / k_{\mathrm{eff}}\right)_{\mathrm{B}}^{\text {calc }}}$, the bias being between the control rod calibration value $\left(\Delta k_{\mathrm{eff}} / k_{\mathrm{eff}}\right)_{\mathrm{B}}^{\mathrm{exp}}$ and the calculated standard perturbation expression

$$
\left.\left(\Delta k_{\text {eff }} / k_{\text {eff }}\right)_{\mathrm{B}}^{\text {calc }}:\left(\frac{\Delta k_{\text {eff }}}{k_{\text {eff }}}\right)_{\mathrm{B}}^{\text {calc }}=\frac{\left\langle\Phi_{0}^{*}, \delta \mathbf{M}_{\mathrm{B}} \boldsymbol{\Phi}_{0}\right\rangle}{\left\langle\boldsymbol{\Phi}_{0}^{*}, k_{\text {eff }}^{-1} \hat{\chi} \mathbf{S}_{\text {fis }, 0} \boldsymbol{\Phi}_{0}\right\rangle}\right) .
$$

Here $\boldsymbol{\Phi}_{0}^{*}$ is an unperturbed standard adjoint flux, and $\delta \mathbf{M}_{\mathrm{B}}=\delta \mathbf{A}_{\mathrm{B}}+\hat{\chi} \delta \mathbf{S}_{\mathrm{fis}, \mathrm{B}}$ is the perturbation of the diffusion (transport) operator related to the control rod insertion (quantity $\rho_{\text {gen }}^{\exp }$ given by Eq. (6) could be obtained, and even more accurately, experimentally via the control rod calibration under subcritical conditions by measuring the $k_{\text {sub }}$ multiplication factor vs. control rod insertion via pulsed source or source jerk techniques [13]).

Likewise, source reactivity $\rho_{\text {source }}^{\text {exp }}$ associated with a given measured external neutron source change $\delta \mathbf{S}_{n}^{\exp }$ may be written as $\rho_{\text {source }}^{\exp }=\frac{\left\langle\Psi^{*}, \delta \mathbf{S}_{n}^{\exp }\right\rangle}{\left\langle\Psi^{*}, \hat{\chi} \mathbf{S}_{\text {fis }, 0} \boldsymbol{\Phi}_{0}\right\rangle}$.

Recalling the definition of importance and assuming that a source perturbation corresponds to a (measured) fractional change of its strength, written as $\delta S_{n}^{\exp } / S_{n, 0}$, we can express $\rho_{\text {source }}^{\exp }$ in terms of the subcritical multiplication factor:

$$
\rho_{\text {source }}^{\exp }=\frac{\delta S_{n}^{\exp }}{S_{n, 0}} \frac{1}{\left\langle\boldsymbol{\Psi}^{*}, \hat{\chi} \mathbf{S}_{\text {fis }} \mathbf{\Phi}_{0}\right\rangle} \equiv \frac{\delta S_{n}^{\exp }}{S_{n, 0}} \frac{1-k_{\text {sub }}}{k_{\text {sub }}} .
$$

If changes in the dedicated control rod and the fusion neutron source do not affect the fission power level, the following relation holds, considering Eqs. (3) and (4):

$$
\rho_{\text {gen }}^{\exp }+\rho_{\text {source }}^{\exp }=0,
$$

and indicates the compensation of the experimental source reactivity and experimental generalized reactivity. Substituting expression (7), we finally obtain a «working» expression for the subcritical multiplication factor:

$$
k_{\text {sub }}=\frac{\delta S_{n}^{\exp } / S_{n, 0}}{\delta S_{n}^{\exp } / S_{n, 0}-\rho_{\text {gen }}^{\exp }} .
$$

Note that $\delta S_{n}^{\exp } / S_{n, 0}$ and $\rho_{\text {gen }}^{\text {exp }}$ have opposite signs.

Following the PCSM method, Eq. (9) can be evaluated by adjusting the neutron source intensity to compensate the control rod insertion. If the online monitoring of this subcriticality index is done with small and slow variations of the source strength, the fission power level will remain practically unaffected.

\section{NUMERICAL VERIFICATION OF THE PCSM METHOD}

A numerical calculation is carried out to verify the PCSM accuracy, assuming that the external neutron source can be modulated to reset the fission power, as required by the procedure [see Eq. (8)]. The calculation is 
performed using the ERANOS code [9] and modeling the fission core in such a way as to make it representative of the TRIGA-RC1 research reactor [10] run at the ENEA-Casaccia Research Center.

The TRIGA-RC1 reactor. The TRIGA-RC1 reactor is a thermal pool research reactor, based on the General Atomic TRIGA Mark II reactor design, operating at a thermal power of 1 MW [10]. The core, in the actual configuration, is a cylindrical system including 111 fuel elements, control rods and an Am-Be-source. Water inside the vessel occupies about $1 / 3$ of the core volume and acts as the first biological shield, neutron moderator and coolant. The RC1-core, surrounded by a graphite reflector, consists of a lattice of TRIGA stainless steel standard fuel elements, graphite dummy elements, as well as control and regulating rods. There are 127 channels on the upper grid plate available for those core components, while the grid itself is divided into seven concentric rings. One channel houses a start-up source (Am-Be), and two fixed channels (the central one and a rabbit) are available for irradiation. A TRIGA fuel element is made of a $\mathrm{HZr}-\mathrm{U}$ ternary alloy, containing $\mathrm{H}$ and $\mathrm{Zr}$ atoms in a ratio of 1.7 to 1 . Due to this ratio, the alloy's crystal lattice has a high level of stability from the metallurgical and nuclear points of view [13]. The fuel is uranium, of which $20 \mathrm{w} / \mathrm{o}$ is ${ }^{235} \mathrm{U}$. Neutron moderation is therefore provided not only by the water coolant, but also by zirconium hydride contained in the alloy, the result being a high-temperature-ready negative coefficient (Doppler effect); and a reactor, which is intrinsically safe. The fuel element is clad in a $0.5 \mathrm{~mm}$ thick stainless steel (AISI 304) tube with graphite cylinders (axial reflectors) at the ends. The fuel element is provided extraneously with two fittings to allow for remotely controlled movements and correct placement into the grid plates.

The reactor power is controlled by a fine regulation rod and three fuel follower rods, two shim rods and a safety rod. The control rods are made from boron carbide, a strong neutron absorber, and have a stainless steel coating.

A cylindrical graphite structure around the core acts as the reactor's lateral reflector. Surrounding this external reflector is a lead thermal shield for gamma rays from the core, and a concrete wall of an average thickness of $2.2 \mathrm{~m}$ serves as a biological shielding.

Thermal power is removed from the core by natural convection and released to the environment through two thermo-hydraulic loops, coupled by two heat exchangers and two cooling towers.

The horizontal section of the core surrounded by the graphite reflector, and the components of the core system, including the fuel elements, control rods and graphite dummy elements. are shown in Fig. 1.
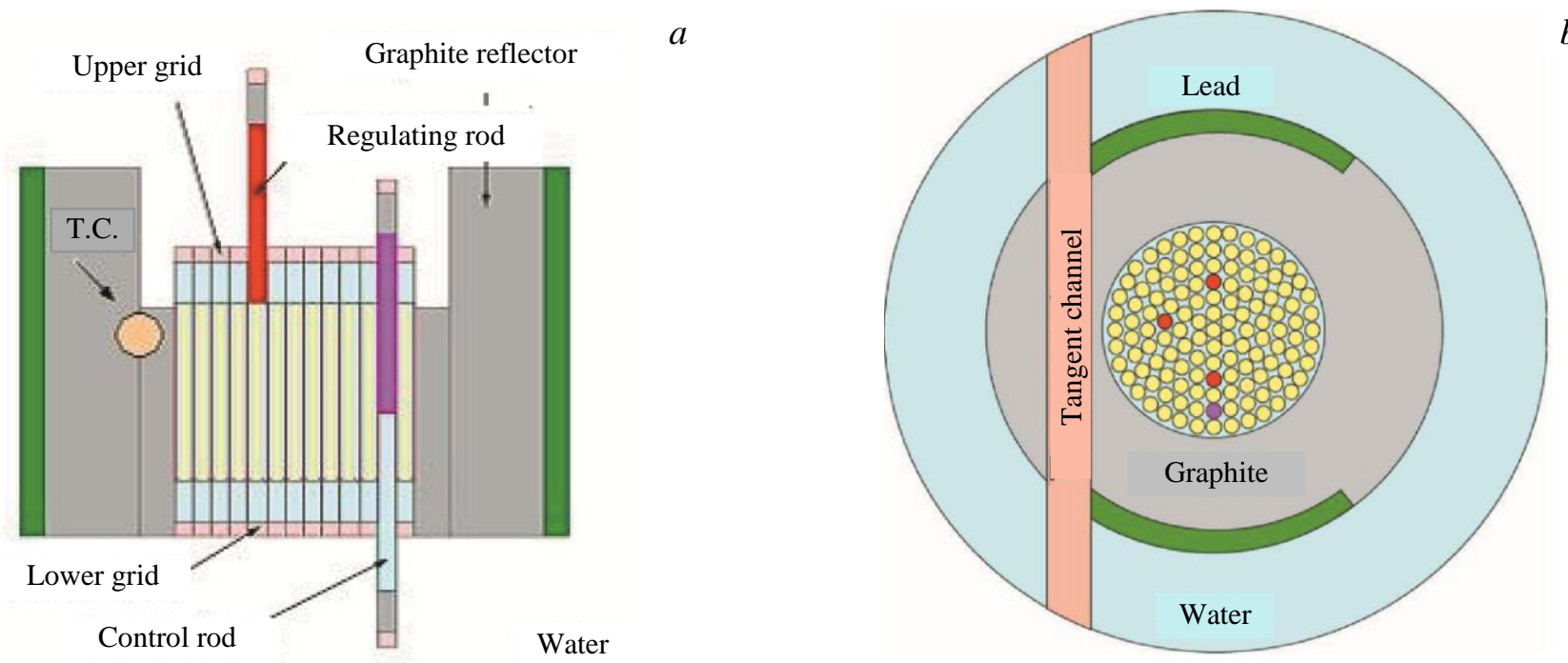

Fig. 1. The core of the TRIGA reactor, vertical section $(a)$, standard configuration $(b)$

Calculation model. For the TRIGA-RC1 model, neutronic calculations were done with the ERANOS-2.2 deterministic code [9]. The JEFF-3.1 nuclear data library [14] has been adopted in the lattice cell calculations for collapsing the fine energy group cross-section structure into one of 33 groups, to be used for the Sn transport calculations of the direct flux and the importance functions. A bi-dimensional RZ-geometry was selected.

Prior to setting up the TRIGA-RC1 model, a study was conducted to investigate the influence of the reactor heterogeneity on its fundamental parameters. The model of choice was a simplified cylindrical model composed of eight zones. They are, starting from the core center, the central channel (labelled as SA), six shells with different fuel compositions (labelled as SB, SC, SD, SE, SF, SG) and the external graphite reflector (labelled as 
$\mathrm{SH})$. The axial and radial sections of the model are shown in Fig. 2, where the central channel is represented in white, the fuel in red and the graphite reflectors in gray.
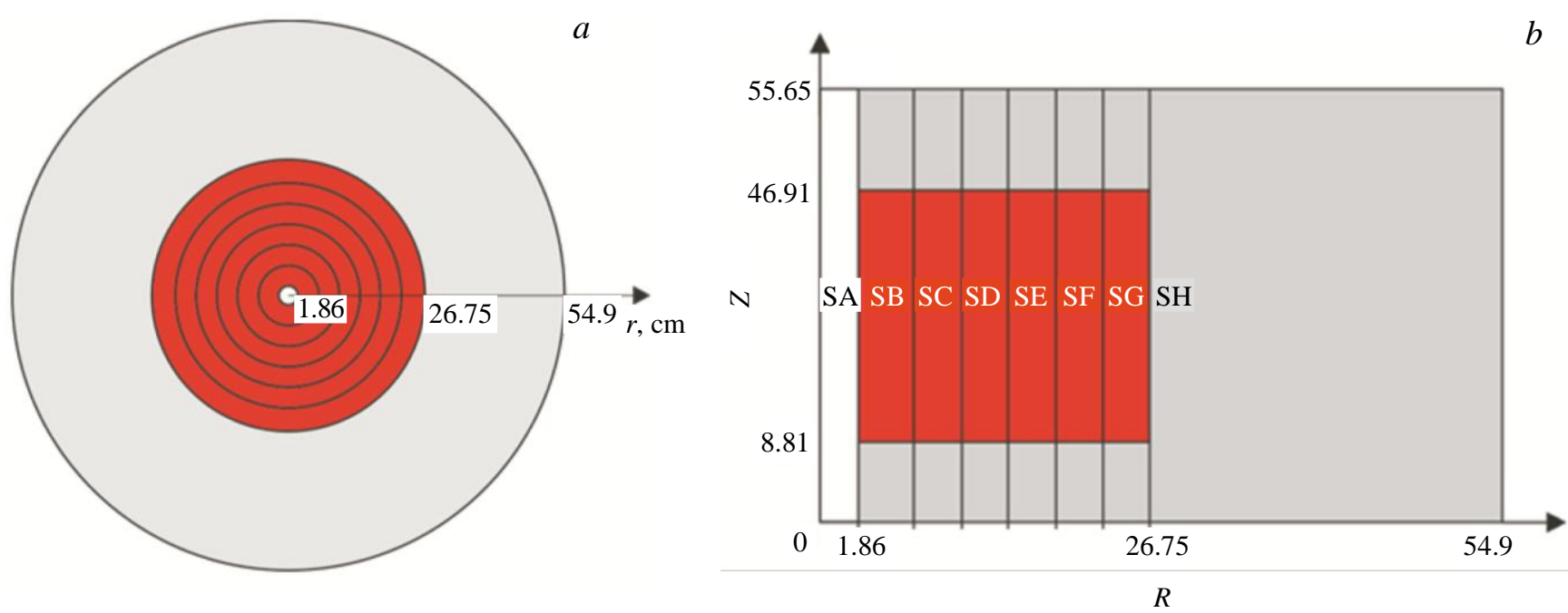

Fig. 2. Sections of the TRIGA reactor core. where the central channel is marked in white, the fuel in red and the graphite reflectors, in gray, radial section $(a)$, axial section $(b)$

Reference and perturbed configurations. Once the TRIGA critical model is created, it is necessary, for the PCSM applicability purposes, to define two different subcritical configurations. a reference one and a perturbed one.

The subcritical reference configuration used for a PCSM-based analysis is derived from the critical one by moving the control rods and involves a core of $k_{\text {eff }}=0.97083$.

The reference configuration is perturbed by introducing a reactivity variation, $\Delta \rho=-0.00866$, by properly moving the control rods to arrive at $k_{\text {eff }}=0.96272$. Although the relative control rods height between the

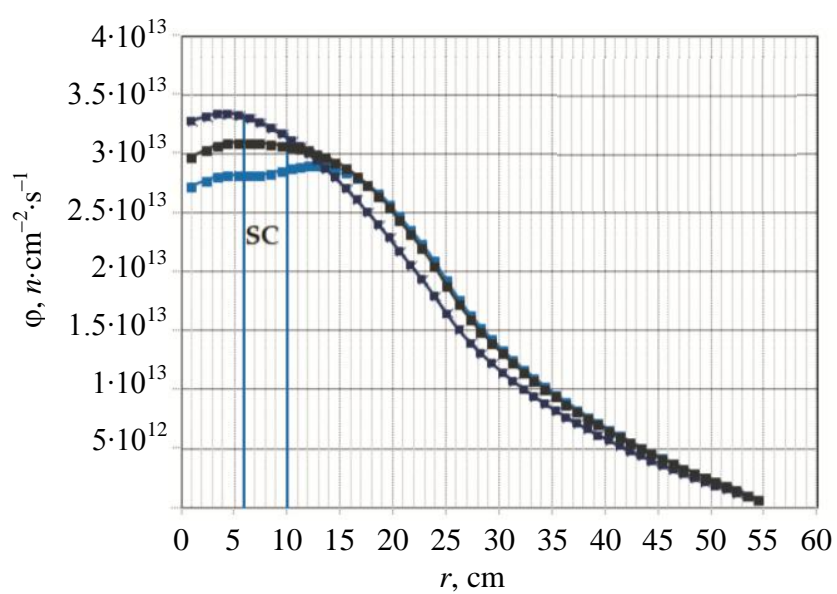

Fig. 3. Critical vs reference vs perturbed configuration, total flux radial traverse at core mid-plane, $k: \square-0.96, \square-1, \mathbf{\square}-0.97$ discussed two subcritical configurations is small, the absorber's effect on the neutron flux is pronounced due to approximations introduced in the control rod model. In fact, the control rods were modeled as a homogenized region in the SC zone, and thus affected a large part of the core, a configuration that differst from the original one, in which the rods are at specific positions relative to the core.

In Fig. 3, we present a homogeneous flux relevant to the three configuration cases described above (critical, reference subcritical and perturbed subcritical). The alteration of the flux shape due to the neutron absorber is evident when passing from the critical configuration to the reference and the perturbed ones.

The adjoint flux and the importance function. The PCSM verification calls for evaluating both the adjoint flux and the importance function the latter being associated with normalized fission power (the «adjoint source»). From the neutronics point of view, the importance function satisfies the inhomogeneous equation derived from the adjoint flux homogeneous equation by adding the adjoint source.

A test subcritical configuration was considered as a starting point for comparing the trends of the importance and the standard adjoint flux functions. They are almost equal when the system is close to criticality and become more and more different as the system deviates from criticality. To investigate this behavior, we 
insert the control rods at an axial height corresponding to $k_{\text {eff }}=0.99048$ (a quasi-critical level). The calculation results are shown in Fig. 4.

The geometry was then modified by displacing the control rods to obtain $k_{\text {eff }}=0.96$. The calculation results are shown Fig. 5.

External neutron source. If the average power of the TRIGA-RC1 reactor in the critical state is about $1 \mathrm{MW}$, the external source is expected to produce the same power in the subcritical state (in accordance with a multiplication factor of 0.97063 , obtained by positioning the control rods at the central channel in an axial position, $z=34.29 \mathrm{~cm}$ ). The source intensity is determined by calculating fission rates corresponding to a given power. The fission rate corresponding to a power of $1 \mathrm{MW}$, $S_{0}=1.091 \times 10^{14} \mathrm{n} / \mathrm{s}$. The source intensity is given in a 33-energy-group structure.

The reference case has then been perturbed by moving the control rods in the axial position, $z=32.28 \mathrm{~cm}$, to decrease reactivity by about 0.00866 $\left(k_{\text {eff }}=0.96272\right)$. In this case, the external source intensity, modified in order to re-establish the initial fission rate, has integral value, $S_{1}=1.508 \times 10^{14} \mathrm{n} / \mathrm{s}$, reflecting a $38 \%$ increase.

The subcritical multiplication factor. The neutronic calculation described above provides all the information required for the verification of the PCSM method: the direct flux, the adjoint flux and the importance function for the reference configuration; the perturbation of the Boltzmann operator that leads to the perturbed subcritical configuration; the external source in the reference configuration, and its modified strength in the perturbed configuration, needed to keep the fission power unaltered.

The generalized reactivity Eq. (5) was calculated using the HGPT methodology, and the system's subcritical reactivity Eq. (9) was obtained. The results are summarized below. Multiplication coefficient $k_{\text {sub: }}$ :
Method ...
PCSM ...
$k_{\text {sub }}$
0.97188
0.97083
0.108
Difference, $\%$...

As one can see, the uncertainty in measuring the discrepancy between the multiplication factors resulting from the PCSM numerical implementation of the PCSM procedure and corresponding to reference conditions is within $0.1 \%$.

This shows how the proposed methodology can be effectively used to determine the subcriticality level of a subcritical reactor without significantly interfering with its normal operation. In summary, steps involved in the PCSM procedure are as follows: (1) preliminary calibration of the dedicated control rod, where a relationship between the rod position change and the corresponding reactivity alteration is established; (2) during operation, a small, slow insertion of the control rod, parallel with the adjustment of the «external» source intensity to ensure that the ex-core and in-core neutron detector readings (proportional to the fission power level) are kept constant; (3) the determination of generalized reactivity, Eq. (5), and $k_{\text {sub }}$ in accordance with Eq. (9). 


\section{FUSION NEUTRON SOURCE MODULATION}

For a fusion-fission hybrid system, the «external» neutron source is generated in a confined plasma, and the PCSM applicability depends on the modulation of the fusion neutron source intensity, intended to reset the fission power.

Several options for modulating the power of a tokamak-confined plasma (and hence changing the fusion neutron source) can be considered. Here, we focus on a method based on plasma compression/expansion. A calculation is therefore carried out to determine the level of plasma compression required to reset the fission power, and in particular the altered values of plasma density and temperature. Depending on adopted compression scheme, a compressed plasma may get out of the toroidal force equilibrium. In that case, an additional calculation is required to determine how the confining magnetic fields should be modified to restore the balance.

Hybrid system specification. The modeling of the hybrid neutronic system is based on a large tokamak, a device with an intermediate toroidal magnetic field operated with $\mathrm{D}-\mathrm{T}$-fuel and including a fission core zone in

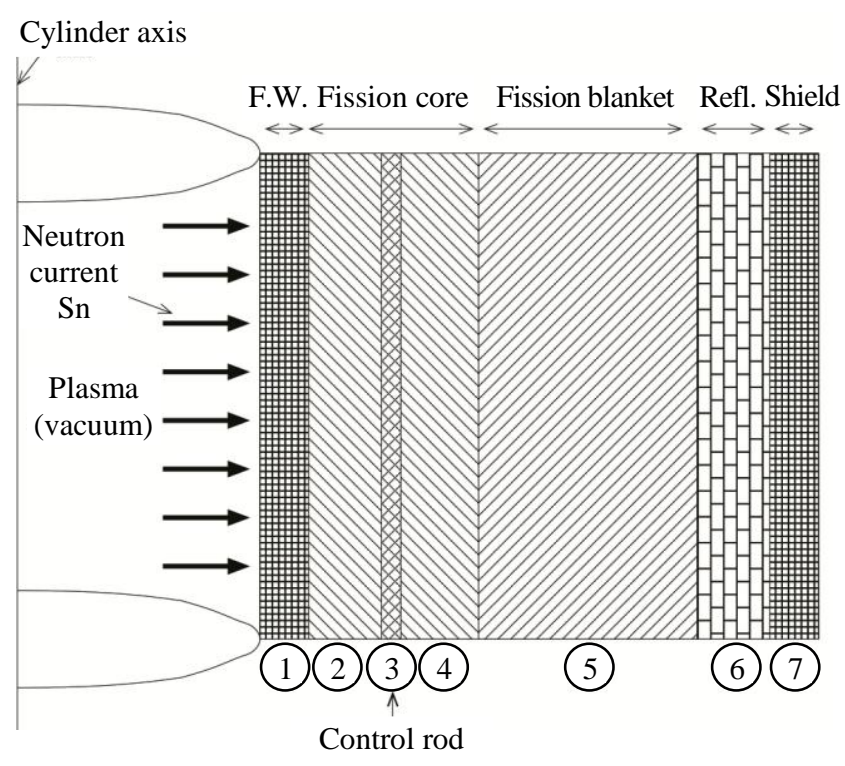

Fig. 6. 1D-modeling of the hybrid system a region between the first wall and the tritium generating blanket. To simplify the neutronic calculations, we have modeled a system with an infinite cylinder (a 1D approximation) with a vacuum cavity at the center and seven homogeneous shells, as shown in Fig. 6.

The central cavity corresponds to the plasma region, where $\mathrm{D}$-T-fusion reactions generate neutrons (the «external» source). The first zone is the tokamak's first wall and vacuum chamber. Zones 2, 3 and 4 constitute the fission core, with zone 3 representing the dedicated control rod. Zone 5 is the fusion (or tritium producing) blanket, followed by the reflector and the shield. The radial distance of the leftmost boundary of zones 1, 2, 5, 6 and 7 are located, respectively, at $R=485,498,535,609$ and $622 \mathrm{~cm}$. The cylinder terminates at $R=633 \mathrm{~cm}$.

Since the objective of this study is to verify the applicability of the PCSM method to a hybrid system, the details of the material composition of the various zones are not particularly relevant, as long as they are representative of a hybrid system. We therefore omit them and only specify the type of materials selected for each different zone. Zone 1 (the first wall) contains iron as a structural material and water as a coolant. Zones 2 and 4 comprising the fission core contain a homogeneous mixture of materials typical of a fast reactor core with the MOX fuel: $\mathrm{UO}_{2}, \mathrm{PuO}_{2}, \mathrm{AmO}_{2}$, plus iron as a structural material and lead as a coolant. The volume fractions for these materials are (the reference configuration): $V_{\mathrm{f}, \mathrm{Pb}}=0.15, V_{\mathrm{f}, \mathrm{Fe}}=0.05, V_{\mathrm{f}, \mathrm{UO}_{2}}=0.3509$, $V_{\mathrm{f}, \mathrm{PaO}_{2}}=V_{\mathrm{f}}, \mathrm{AmO}_{2}=0.2245$. Zone 3 has the same composition as zones 2 and 4 when the control rod is extracted (the reference configuration), and a modified composition, in which boron-10 replaces part of the fuel, when the control rod is partially inserted (the perturbed configuration). Zone 5 (the fusion blanket) contains lithium and lead, the latter acting both as a multiplier and a coolant medium. Zone 6 (the reflector) is made of carbon and water, while the last zone is a shield with the same material composition as zone 1 .

Fusion plasma's sub-ignited reference state. The reference configuration of the hybrid system consists of a plasma operating in a sub-ignited steady state during some form of auxiliary heating, and a subcritical fission core $\left(k_{\text {eff }} \sim 0.97\right)$. To specify the plasma state, and in particular its working temperature, we consider its energy balance. After that, we can determine the fusion neutron source which maintains the fission core subcriticality.

Plasma operating regime. In the reference configuration, we assume that a $\mathrm{D}-\mathrm{T}$-plasma (50/50 deuterium/tritium) of a $1.1 \times 10^{20} 1 / \mathrm{m}^{3}$ density is used. The tokamak major radius, $R_{\mathrm{ax}}=R_{0}=350 \mathrm{~cm}$, the horizontal minor radius, $a=120 \mathrm{~cm}$, the elongation of the plasma cross-section, $\kappa=b / a=1.74$. As a result, plasma volume, 
$V_{\mathrm{pl}}=2 \pi \mathrm{a}^{2} \kappa=1.74 \mathrm{~m}^{3}$. The on-axis toroidal magnetic field, $B_{\mathrm{t}}=5.5 \mathrm{~T}$, the safety factor on the plasma edge, $q^{*}=3.5$, and the total plasma current at flat-top is set equal to $I_{\mathrm{pl}}=11 \mathrm{MA}$.

For simplicity, we consider the 0-D-equation for the time evolution of plasma thermal energy [15]:

$$
\frac{d W(t)}{d t}=\left\langle S_{\alpha}\right\rangle+\left\langle S_{\Omega}\right\rangle+S_{\text {aux }}-\left\langle S_{\mathrm{B}}\right\rangle-\left\langle S_{\mathrm{tr}}\right\rangle \quad\left[\mathrm{W} / \mathrm{m}^{3}\right],
$$

where plasma internal energy, $W=\langle 3 n T\rangle$, fission power carried by alpha particles, $S_{\alpha}=\left\langle E_{\alpha} n^{2}\langle\sigma v\rangle / 4\right\rangle$, ohmic heating power, $S_{\Omega}=\left\langle\eta J^{2}\right\rangle$, and auxiliary heating power, $S_{\text {aux }}$, the Bremsstrahlung and transport power losses, $S_{\mathrm{B}}=\left\langle C_{\mathrm{B}} n^{2} T^{1 / 2}\right\rangle$ and $S_{\mathrm{tr}}=\left\langle 3 n T / \tau_{E}\right\rangle$, are given in MKSA units by: $W(t)=5.34 \times 10^{4} \bar{n}_{20} \bar{T}_{k}(t)$, $\left\langle S_{\alpha}\right\rangle=2.31 \times 10^{5} \bar{n}_{20}^{2}\langle\sigma v\rangle_{n},\left\langle S_{\Omega}\right\rangle=\frac{1}{V_{\mathrm{pl}}} \frac{5.6 \times 10^{4}}{1-1.31 \varepsilon^{1 / 2}+0.46 \varepsilon} \frac{I_{\mathrm{pl}, M}^{2} R_{0}}{a^{2} \kappa \bar{T}_{k}^{3 / 2}(t)}, \quad S_{\text {aux }}=$ given function of $\bar{T}_{k}(t)$, $\left\langle S_{\mathrm{B}}\right\rangle=6.14 \times 10^{3} \bar{n}_{20}^{2} \bar{T}_{k}^{1 / 2}(t),\left\langle S_{\text {tr }}\right\rangle=5.34 \times 10^{4} \frac{\bar{n}_{20} \bar{T}_{k}(t)}{\tau_{E}}$.

In the above equations, $\langle\mathrm{L}\rangle$ denotes averaging over the plasma volume, the over-bar indicates averaging over the plasma cross-section, $\bar{n}_{20}=\bar{n} / 10^{20}$ is the particle density in $1 / \mathrm{m}^{3}$ divided by $10^{20}, \varepsilon=a / R_{0}$ is the inverse aspect ratio, $I_{\mathrm{pl}, M}$ is the total plasma current in MA, and $\langle\sigma v\rangle_{n}=\langle\sigma v\rangle / 10^{-22}$, where the reactivity $\langle\sigma v\rangle=10^{-6} \exp \left[-21.38 / T_{k}^{0.2935}-25.20-7.101 \times 10^{-2} T_{k}+1.938 \times 10^{-4} T_{k}^{2}+4.925 \times 10^{-6} T_{k}^{3}-3.984 \times 10^{-8} T_{k}^{4}\right]$ is expressed in $\mathrm{m}^{3} / \mathrm{s}$, when temperature is in $\mathrm{keV}$.

For simplicity, we assume plasma confinement time, $\tau_{E}=1.2 \mathrm{~s}$, to be constant. This is a rather crude approximation, as the plasma regime is sustained by auxiliary heating. However we use this assumption, considering the demonstration nature of our study. The various powers in the plasma temperature function are shown in Fig. 7.

The vertical lines indicate two important temperatures characterizing a magnetized fusion plasma: the Bremsstrahlung temperature, defined by balance $\left\langle S_{\alpha}\right\rangle+\left\langle S_{\Omega}\right\rangle=\left\langle S_{\mathrm{B}}\right\rangle$, and the ignition temperature, defined by $\left\langle S_{\alpha}\right\rangle+\left\langle S_{\Omega}\right\rangle=\left\langle S_{\mathrm{B}}\right\rangle+\left\langle\mathrm{S}_{\mathrm{t}}\right\rangle$. We find $T_{\mathrm{B}}=3.512 \mathrm{keV}$ and $T_{1}=15.400 \mathrm{keV}$, respectively.

We solved Eq. (10) modeling the auxiliary heating function to obtain a steady-state sub-ignited regime

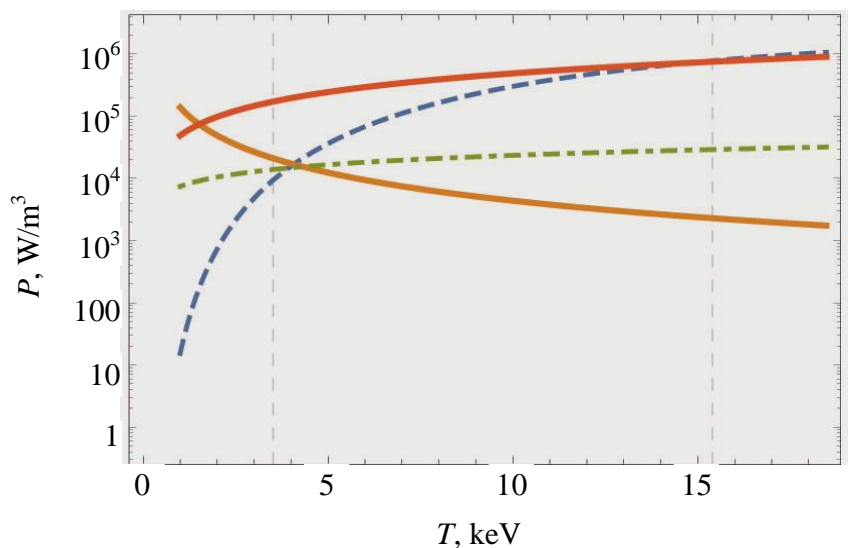

Fig. 7. Power components contributing to the plasma energy balance. Vertical lines indicate Bremmstrahlung and ignition temperatures. Bremmstrahlung and transport powers are multiplied by -1 for ease of comparison: $----+\mathrm{FUS},--$ $+\mathrm{CHM},-=----\mathrm{BR},---\mathrm{TR}$ with working temperature, $T_{\text {sub }}=9 \mathrm{keV}$. In our simulation, temperature $0.95 T_{\text {sub }}$ is reached in about $16.2 \mathrm{~s}$, and the steady-state is sustained by auxiliary heating, $S_{\text {aus }}=0.228 \times 10^{6} \mathrm{~W} / \mathrm{m}^{3}$.

Plasma neutron source. The fusion neutron source depends on the rate of the fusion reaction in plasma, which in turn depends on plasma temperature and density. Plasma eccentricity, $e \equiv \sqrt{1-1 / \kappa^{2}}=0.821$. The fusion reaction rate, $R_{\text {fus }}=n^{2}\langle\sigma \mathrm{v}\rangle / 4$ in $1 /\left(\mathrm{m}^{3} \cdot \mathrm{s}\right)$, and is also equal to the number of fusion neutrons produced per unit plasma volume per second, $n_{\mathrm{f}}$. In the reference sub-ignited plasma configuration with $T_{k}=9 \mathrm{keV}$, we find $n_{f}=2.483 \times 10^{11} 1 /\left(\mathrm{cm}^{3} \cdot \mathrm{s}\right)$. Introducing factor $f_{\mathrm{b}}$ denoting the fraction of the plasma which is burning, that is, actually undergoing an appreciable number of fusion reactions, we obtain the total number (rate) of fusion neutrons produced in the plasma per second, $N_{f}=n_{f} V_{\mathrm{p} f} f_{\mathrm{b}}=8.647 \times 10^{18} 1 / \mathrm{s}$.

We then evaluate the density of the current of fusion neutrons impinging on the first wall. The latter, with its horizontal and vertical minor radii denoted by $a_{\mathrm{w}}$ and $b_{\mathrm{w}}$, respectively, is assumed to be $15 \mathrm{~cm}$ away from the 
plasma boundary. Its total surface, $S_{\mathrm{w}}=8 \pi b_{\mathrm{w}} R_{0} E\left(e_{\mathrm{w}}\right)=2.419 \times 10^{6} \mathrm{~cm}^{2}$, where $E$ denotes the complete elliptic integral, and $e_{\mathrm{w}}=\sqrt{1-1 / \kappa_{\mathrm{w}}^{2}}$, with $\kappa_{\mathrm{w}}=b_{\mathrm{w}} / a_{\mathrm{w}}$. The fusion neutron current hitting the outer part of the first wall is

$$
J_{\mathrm{pl}, 0}=\frac{N_{\mathrm{f}}}{S_{\mathrm{w}}}=\frac{\pi}{16} \frac{n^{2}\langle\sigma v\rangle(T) a^{2} f_{\mathrm{b}}}{a_{\mathrm{w}} E\left(e_{\mathrm{w}}\right)}=3.574 \times 10^{12} 1 /\left(\mathrm{cm}^{2} \cdot \mathrm{s}\right) .
$$

Fission core reference subcritical state. Neutronic calculations for the reference and perturbed configurations of the hybrid system were carried out using a 1D-code based on a finite-difference numerical technique, writen specifically for this study. It allows solving multi-group diffusion equation with and without an external source. It calculates both the direct and adjoint fluxes, as well as relevant importance functions. The code has been benchmarked against analytical solutions of the 2-group, 2-zone problem, while a more throughout validation work using the ERANOS code is underway.

We have discretized the radial dimension in 45 grid points, and chosen a 6-group scheme. The group upper energies are $20 \times 10^{6}, 4.5 \times 10^{6}, 0.82 \times 10^{6}, 0.14 \times 10^{6}, 3.71 \times 10^{3}, 3.06 \mathrm{eV}$, with the fission spectrum given by $\chi=(0.18,0.655,0.215,0.012,0,0)$. Where needed, the high-energy neutron source is introduced, but only in the high-energy group $(g=1)$ equation. The neutronic group constants were prepared by processing ENDF/B data with the NJOY code, using a fusion-dedicated weighting spectrum. The solution procedure is the conventional power iteration method. In all computations, we used convergence tolerances for the multiplication factor and the flux ( $\epsilon_{k}=10^{-5}$ and $\epsilon_{\varphi}=10^{-4}$, respectively).

The neutronic calculation performed for this model of a hybrid system are identical to those outlined in the «Numerical verification of the PCSM method» section in respect of the TRIGA reactor model, and are only summarized here in the form of key verification results. The subcritical reference configuration has $k_{\text {eff }}=0.97001$, and is maintained in steady state by the fusion neutron current density given in Eq. (11), while generating a fission power of $W_{0}=2.307 \times 10^{5} \mathrm{~W}$. The perturbed configuration, on the other hand, has $k_{\mathrm{eff}}=0.96$, and generates a fission power of $1.814 \times 10^{5} \mathrm{~W}$ with the same fusion neutron source as the one used in the reference configuration. To reset the reference fission power $W_{0}$, the fusion plasma current $J_{\mathrm{pl}, 1}$ needs to be increased to $5.128 \times 10^{12} 1 /\left(\mathrm{cm}^{2} \cdot \mathrm{s}\right)$.

To produce a higher fusion neutron current, plasma density $n$ and temperature $T$ must be changed accordingly. Using Eq. (11), the condition to be satisfied by the new plasma parameters is (the subscript «1» stands for final state)

$$
\frac{n_{1}^{2}\langle\sigma v\rangle\left(T_{1}\right) a_{1}^{2} f_{\mathrm{b}}}{a_{\mathrm{w}} E\left(e_{\mathrm{w}}\right)}=\frac{16}{\pi} J_{\mathrm{pl}, 1}=2.612 \times 10^{13},
$$

with density indicated in $1 / \mathrm{cm}^{3}$, temperature in $\mathrm{keV}$, and $\langle\sigma v\rangle\left(T_{1}\right)$ in $\mathrm{cm}^{3} / \mathrm{s}$, and where $a_{1}$ and $a_{\mathrm{w}}$ denote the minor radius of the compressed plasma and of the first wall.

Fusion neutron source modulation by plasma compression/expansion. Finally, it is necessary to consider a method for increasing the fusion neutron source strength, as required by the PCSM procedure. We expect the required fusion power modulation to be achieved through a continuous series of plasma quasi-static compressions/expansions, induced by appropriate changes of the confining toroidal and/or vertical magnetic field. Ultimately, this is accomplished by simply varying the currents flowing in the toroidal field coils (say, $I_{\mathrm{TFC}}$ ) and/or poloidal field coils $\left(I_{\mathrm{PFC}}\right)$. In general, an $I_{\mathrm{TFC}}$ increase amplifies the toroidal magnetic field and reduces the plasma minor radius (invariance of the toroidal magnetic flux). A $I_{\mathrm{PFC}}$ increase on the contrary, raises the inward force acting on the plasma, which, in accordance with the toroidal force balance, counteracts the outward-directed forces associated with the pressure and the magnetic field (the toroidal effects) [15]. If the plasma is in toroidal equilibrium, an increase in $I_{\mathrm{PFC}}$ displaces the plasma inward toward a new equilibrium position. If it is out of equilibrium, an appropriate change in $I_{\mathrm{PFC}}$ can restore the equilibrium without the need for any displacement. Note that a plasma displaced inward encounters a stronger toroidal field $\left(B_{\text {tor }} \propto 1 / R\right)$, and becomes compressed [16]. Adiabatic plasma compression experiments that have been carried out since the 1970s have proved the effectiveness of this heating technique $[17,18]$. 
The scaling laws for the magnetic compression were discussed in [19] with the assumption that plasma is perfectly conductive (the «frozen-in» plasma compression). These scaling laws are derived from three basic lowest order constraints, which apply when a plasma is compressed slowly in relation to particle collision time $\tau_{\mathrm{p}}$ («collisional» compression: time $\tau_{\mathrm{c}}<\tau_{\mathrm{p}}$ ), but rapidly in relation to energy confinement time $\tau_{E}$ («adiabatic» compression time $\tau_{\mathrm{c}}>\tau_{E}$ ). These are the total number of particles inside a magnetic surface, the toroidal magnetic flux inside a magnetic surface, and the rotational transform (equivalently, the poloidal flux). In a zerodimensional approximation, these constraints, augmented by the adiabatic energy law, read $r^{2} R_{\mathrm{ax}} n=\mathrm{const}$, $r^{2} B_{\mathrm{t}}=$ const, $q=\frac{r B_{\mathrm{t}}}{R_{\mathrm{ax}} B_{\mathrm{p}}}=$ const, $\frac{T}{n^{2 / 3}}=$ const, where $n, B_{\mathrm{t}}, B_{\mathrm{p}}, q$ indicate the density, the toroidal and poloidal magnetic fields, and the safety factor (inverse of the rotational transform), respectively, $r$ localizes the magnetic surface, and $R_{\mathrm{ax}}$ denotes the major radius. Assuming that $r=a$, we obtain the following general compression scaling laws [19]:

$$
\begin{gathered}
n=\frac{\text { const }}{a^{2} R_{\mathrm{ax}}} ; \quad T=\frac{\text { const }}{a^{4 / 3} R_{\mathrm{ax}}^{2 / 3}} ; \quad I_{\mathrm{pl}}=\frac{\text { const }}{R_{\mathrm{ax}}} ; \\
B_{\mathrm{t}}\left(R_{\mathrm{ax}}\right)=\frac{\text { const }}{a^{2}} ; \quad B_{\mathrm{p}}=\frac{\text { const }}{a R_{\mathrm{ax}}} .
\end{gathered}
$$

A scaling law will also be needed for the plasma inductance. Neglecting plasma resistivity, we assume [20]:

$$
L_{\mathrm{pl}} I_{\mathrm{pl}} ; \text { const } \Rightarrow L_{\mathrm{pl}}=\text { const } R_{\mathrm{a}} \text {. }
$$

Here $L_{\mathrm{pl}}$ is the total inductance of the plasma, which comprises both the internal and the external contributions: $L_{\mathrm{pl}}=L_{\text {in }}+L_{\mathrm{ext}}$.

Whareas in [19] three different compression types are described, here we consider only two of them, namely, those denoted in [19] as «R-and- $a$ compression, type $\mathrm{B} »$ and «Constant- $R$ a-compression».

Compression based on inward plasma displacement. In this compression mode (the « $R$-and- $a$-compression, type B» sketched in Fig. 8), the external vertical field increases (due to the $I_{\mathrm{PFC}}$ increase) displacing plasma radially inward and thereby inducing a compression in the minor radius due to increased toroidal field. No change in $I_{\mathrm{TFC}}$ is required. The application of the toroidal flux conservation $a_{1}^{2} B_{t, 1}\left(R_{\mathrm{ax}, 1}\right)=a_{0}^{2} B_{\mathrm{t}, 0}\left(R_{\mathrm{ax}, 0}\right)$, augmented by the $1 / R$ law $B_{\mathrm{t}, 1}\left(R_{\mathrm{ax}, 1}\right)=B_{\mathrm{t}, 0}\left(R_{\mathrm{ax}, 0}\right) R_{\mathrm{ax}, 0} / R_{\mathrm{ax}, 1}$, leads to $\left(a_{1} / a_{0}\right)^{2}=R_{\mathrm{ax}, 1} / R_{\mathrm{ax}, 0}$ and to the compression scaling $a=$ const $R_{\mathrm{ax}}^{1 / 2}$ (as shown in Fig. 8). In [19] this

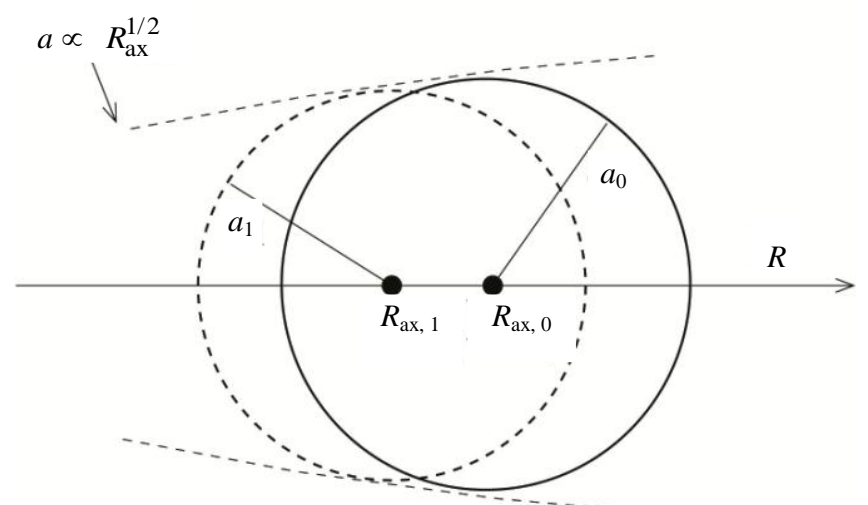

Fig. 8. Sketch of the compression scheme based on inward plasma displacement [19] compression method is proposed as an auxiliary heating mechanism to be used during the discharge startup to heat up the plasma to required temperatures. Large plasma displacements were therefore envisaged, with the consequent need for a greater plasma chamber. In the context of this study, compression is performed with the only goal of inducing relatively small temperature changes, as required by the PCSM, and this practical disadvantage is minimized.

Defining the compression factor, $C_{\mathrm{R}} \equiv R_{\mathrm{ax} 0} / R_{\mathrm{ax} 1}=\left(a_{0} / a_{1}\right)^{2}$, we obtain $B_{\mathrm{t}, 1}\left(R_{\mathrm{ax}, 1}\right)=B_{\mathrm{t}, 0}\left(R_{\mathrm{ax}, 0}\right) C_{\mathrm{R}}$ and, for the aspect ratio, $A_{1}=A_{0} / C_{\mathrm{R}}^{1 / 2}$. The (13)-(15) scalings describing this compression approach are $n_{1}=n_{0} C_{\mathrm{R}}^{2}, T_{1}=T_{0} C_{\mathrm{R}}^{4 / 3}, p_{1}=p_{0} C_{\mathrm{R}}^{10 / 3}, B_{\mathrm{t}, 1}\left(R_{\mathrm{ax}, 1}\right)=B_{\mathrm{t}, 0}\left(R_{\mathrm{ax}, 0}\right) C_{\mathrm{R}}, \quad I_{\mathrm{pl}, 1}=I_{\mathrm{pl}, 0} C_{\mathrm{R}}, q_{1}\left(a_{1}\right)=q_{0}\left(a_{0}\right), B_{\mathrm{p}, 1}\left(a_{1}\right)=B_{\mathrm{p}, 0}\left(a_{0}\right) C_{\mathrm{R}}^{3 / 2}$ and $L_{\mathrm{pl}, 1}=L_{\mathrm{pl}, o} / C_{\mathrm{R}}$. Introducing these scalings in Eq. (12) we obtain the following relation for the required compression parameter $C_{\mathrm{R}}$ :

$$
\frac{C_{\mathrm{R}} n_{0}^{2}\langle\sigma v\rangle\left(T_{0} C_{\mathrm{R}}^{4 / 3}\right) a_{0}^{2} f_{\mathrm{b}}}{a_{\mathrm{w}} E\left(e_{\mathrm{w}}\right)}=2.612 \times 10^{13},
$$


where, as assumed, elongation $\kappa$ is invariant under compression. A numerical solution leads to $C_{R}=1.084$, and consequently $a_{1}=a_{0} / \sqrt{C_{\mathrm{R}}}=1.153 \mathrm{~m}, R_{\mathrm{ax}, 1}=3.230 \mathrm{~m}, n_{1}=1.292 \times 10^{20} 1 / \mathrm{m}^{3}$ and $T_{1}=10.020 \mathrm{keV}$. The increment in the number of fusion neutrons produced in the compressed plasma, $N_{\mathrm{f}, 1} / N_{\mathrm{f}, 0}=C_{\mathrm{R}}^{2}\langle\sigma v\rangle\left(T_{0} C_{\mathrm{R}}^{4 / 3}\right) /\langle\sigma v\rangle\left(T_{0}\right)=1.555$.

We now calculate the change in the vertical magnetic field required to implement the compression described above. The vertical field is necessary in a toroidal configuration to enforce the toroidal force balance, which requires that forces tending to drive the plasma outward are counteracted by an inward Lorentz force induced by a vertical magnetic field $F_{v}$. There are three plasma-expanding forces, namely, the $F_{\mathrm{p}}$ «tire-tube» force, the $F_{1 / R}$ «1/R» force, and the $F_{\text {hoop }}$ «hoop» force: $F_{\mathrm{p}}+F_{1 / R}+F_{\text {hoop }}=F_{v}$. An explicit expression for these four forces in the limit of a circular plasma cross section can be found in [15]. Here we only report the expression for a (externally imposed) vertical magnetic field needed to establish the toroidal equilibrium:

$$
\frac{B_{\mathrm{v}}}{B_{\mathrm{p}}(a)}=\frac{1}{4} \frac{a}{R_{\mathrm{ax}}}\left[\frac{2 \mu_{0} \overline{p(r)}}{B_{\mathrm{p}}(a)^{2}}+\frac{B_{\mathrm{t}}(a)^{2}-\overline{B_{\mathrm{t}}(r)^{2}}}{B_{\mathrm{p}}(a)^{2}}+1+2\right],
$$

where $1=1_{\text {int }}+1_{\text {ext }}$ is the total normalized plasma inductance comprising the internal and the external contributions. In these expressions, $p(r)=n(r) T(r)$ is the plasma pressure, while $B_{\mathrm{t}}, B_{\mathrm{p}}, B_{\mathrm{v}}$ are the toroidal, poloidal (induced by the plasma current), and external vertical magnetic fields. The $B_{\mathrm{t}, \text { ex }}$ field is the vacuum (externally imposed) magnetic field, calculated at $R=R_{\mathrm{ax}}$.

To obtain a numerical value for $B_{\mathrm{v}}$ before and after the compression, we need to specify the corresponding radial dependence of plasma profiles $p(r)=n(r) T(r), B_{\mathrm{t}}(r), B_{\mathrm{p}}(r)$. A good model for the minor radius dependence of the plasma profiles in the reference configuration (i.e., before compression) is provided by the screw-pinch approximation to the tokamak case, which is formally obtained by the torus straightening out into a cylinder. The radial pressure balance for the screw-pinch reads

$$
\frac{d}{d r}\left(p(r)+\frac{B_{\theta}^{2}(r)}{2 \mu_{0}}+\frac{B_{\varphi}^{2}(r)}{2 \mu_{0}}\right)+\frac{B_{\theta}^{2}(r)}{\mu_{0} r}=0 .
$$

Based on experimental results on pinch devices, as well as simplicity considerations, we adopt the following magnetic field components [21]:

$$
\begin{gathered}
B_{\theta}(r, \theta)=B_{\theta}(r) \frac{R_{\mathrm{ax}}}{R(r, \theta)}, \quad \text { where } B_{\theta}(r)=\frac{\mu_{0} I_{\mathrm{pl}}}{\pi} \frac{r}{r^{2}+a^{2}} ; \\
B_{\varphi}(r, \theta, \lambda)=B_{\varphi}(r) \frac{R_{\mathrm{ax}}}{R(r, \theta)}, \quad \text { where } B_{\varphi}^{2}(r)=B_{\varphi, \mathrm{ex}}^{2}-2 \mu_{0} \lambda p(r ; a),
\end{gathered}
$$

where $B_{\varphi, \text { ex }}$ is the externally applied toroidal field (which is a constant, as it does not include the $1 / R$ dependence), and where $0<\lambda<1$ is a parameter quantifying the degree of diamagnetism (reduction of the externally imposed toroidal magnetic field due to the presence of the plasma) - we will take $\lambda=0.50$. In particular, the toroidal field on axis is given by $B_{\varphi}(0,0)^{2}=B_{\varphi, \text { ex }}^{2}-2 \mu_{0} \lambda p(0 ; a)$. Once the magnetic field is defined, Eq. (17) gives the pressure profile: $p(r, a)=\hat{p}(a)\left[4\left(3 a^{4}-2 a^{2} r^{2}-r^{4}\right)\right] /\left[3\left(a^{2}+r^{2}\right)^{2}\right]$, where $\hat{p}(a)=\left(3 \mu_{0} I_{\mathrm{pl}}^{2}\right) /\left[32 \pi^{2} a^{2}(1-\lambda)\right]$ is the value on axis, and $p( \pm a, a)=0$.

With this information at hand, we can find an expression for the vertical magnetic field needed to establish the toroidal equilibrium in the initial (pre-compression) configuration. We obtain

$$
B_{\mathrm{v}, 0}=\frac{\mu_{0} I_{\mathrm{pl}, 0}}{8 \pi R_{\mathrm{ax}, 0}}\left[\frac{1+\lambda}{1-\lambda}+2 \ln \left(\frac{8 R_{\mathrm{ax}, 0}}{a_{0}}\right)-\frac{3}{2}\right],
$$

where the first term inside the square brackets includes the tire-tube force and the $1 / R$ force, and the last two terms come from the hoop force. 
The compression scaling for the pressure is derived from density and temperature scalings. From the generic expressions for the latter two profiles, $n(r)=\hat{n} f_{n}(r ; a)$ and $T(r)=\hat{T} f_{\mathrm{T}}(r ; a)$ respectively [with the $f$ functions becoming 1 at $r=0]$, we obtain $p_{0}(r)=\hat{n}_{0} f_{n}\left(r ; a_{0}\right) \hat{T}_{0} f_{\mathrm{T}}\left(r ; a_{0}\right) \equiv \hat{p}\left(a_{0}\right) f_{p}\left(r ; a_{0}\right)$ and $p_{1}(r)=C_{\mathrm{R}}^{10 / 3} \hat{p}\left(a_{0}\right) f_{p}\left(r ; a_{1}\right)$, where $f_{p}(r ; a)=4\left(3 a^{4}-2 a^{2} r^{2}-r^{4}\right) /\left[3\left(a^{2}+r^{2}\right)^{2}\right]$. From Eq. (18), we finally obtain the scaling for the toroidal magnetic field: $B_{\mathrm{t}, 1}=\left\{C_{\mathrm{R}}^{2} B_{\mathrm{t}, \mathrm{ex}, 0}^{2}-2 \mu_{0} \lambda C_{\mathrm{R}}^{10 / 3} \hat{p}\left(a_{0}\right)\left[4\left(3 a_{1}^{4}-2 a_{1}^{2} r^{2}-r^{4}\right) / 3\left(a_{1}^{2}+r^{2}\right)^{2}\right]\right\}^{1 / 2}$.

Introducing the scaling properties of various quantities in Eq. (16), we obtain the following expression for the required magnetic field after compression: $B_{\mathrm{v}, 1}=\frac{\mu_{0} I_{\mathrm{pl}, 0}}{8 \pi R_{\mathrm{ax}, 0}}\left\{C_{\mathrm{R}}^{7 / 3} \frac{1+\lambda}{1-\lambda}+C_{\mathrm{R}}^{2}\left[2 \ln \left(\frac{8 R_{\mathrm{ax}, 0}}{a_{0}}\right)-\frac{3}{2}\right]\right\}$.

Numerically, we find that the vertical field should be changed from $1.786 \mathrm{~T}$ before the compression, to $2.121 \mathrm{~T}$ after the compression, $a \sim 18.7 \%$ increase.

Compression based on plasma minor radius reduction. We also considered a second compression scheme, the one identified as «Constant-R a-compression» in [19]. In this approach, the toroidal field is increased to a point allowing plasma compression while the vertical field is increased to maintain the toroidal force balance. The plasma major radius remains unchanged during the compression, $R_{\mathrm{ax}, 1}=R_{\mathrm{ax}, 0}$ (no plasma displacement). A convenient parameter to describe this type of compression is the ratio of the plasma radius before and after the compression: $C_{a} \equiv a_{0} / a_{1}>1$. The aspect ratio scaling, $A_{1}=A_{0} C_{a}$.

A procedure similar to the one presented above yields the following results: $C_{a}=1.0678$; plasma radius compression from $a_{0}=120$ to $a_{1}=112.38 \mathrm{~cm}$; new plasma density and temperature $n_{0}=1.100 \times 10^{20} \rightarrow n_{1}=1.254 \times 10^{20} 1 / \mathrm{m}^{3}$ and $T_{0}=9 \rightarrow T_{1}=9,823 \mathrm{keV}$; increment in the total number of fusion neutrons produced in compressed plasma, $N_{f, 1} / N_{f, 0}=1.435$. To maintain the toroidal plasma balance, the vertical magnetic field must be strengthened from $B_{\mathrm{v}, 0}=1.786$ to $B_{\mathrm{v}, 1}=1.859, a \sim 3.6 \%$ increase.

\section{SUMMARY AND CONCLUSIONS}

We investigate the possibility of applying the PCSM (power control-based subcriticality monitoring) to safely determine a fusion-fission hybrid subcriticality without significantly interfering with the normal operation of the fission core, where nuclear waste can be transmuted and/or fissile fuel can be bred. The method consists in: (i) a standard calibration of a dedicated control rod in the fission core - a relationship between a control rod position change and the corresponding reactivity alteration may then be established; (ii) during operation, a small, slow insertion of the control rod and associated adjustment of the fusion neutron source (i.e., fusion power generated in the plasma) to ensure that the ex-core and in-core neutron detector readings are kept constant and the fission power level is unchanged; (iii) an evaluation of the subcritical multiplication factor using Eq. (9).

Neutronic calculations based on the multi-group transport equation and the PCSM procedure allowed the subcritcality evaluation with an accuracy of the order of $0.1 \%$, suggesting that the proposed methodology can be effectively used to determine a hybrid system's subcriticality. The enhancement of the fusion neutron source strength required to reset the fission power following the control rod insertion was accomplished using plasma compression. The application of a simple 0-D-plasma power balance equation alongside a screw-pinch modelization of plasma profiles showed that the compression scheme based on inward displacement of the major radius requires minor radius and vertical magnetic field changes of $-3.9 \%$ and $+18.7 \%$, respectively, while the plasma compression scheme based on a reduced minor radius and a fixed major radius requires minor radius and vertical magnetic field changes of $-6.3 \%$ and $+3.6 \%$, respectively. The size of these changes seems to be acceptable from an operational point of view.

Due to a number of simplifications used in our calculation, especially those regarding the plasma compression modeling, this study is far from providing a convincing evidence that the PCSM coupled with a plasma compression/expansion technique can be implemented in an hybrid system based on the tokamak concept. In particular, the fusion power modulation by compression, required by the PCSM, is a delicate 
endeavor, because the parameters of a magnetically confined plasma, such as density, temperature and pressure must meet strict constraints to avoid instabilities. The latter can cause irregularities in the operation, from anomalously large transport rates, to major disruptions (a sudden loss of confinement). Our simplified approach to fusion power modulation via plasma compression/expansion did not consider the issue of plasma stability.

In addition to plasma stability, several other points remain to be addressed in order to be confident about the suitability of the PCSM method coupled with the plasma compression/expansion technique, adopted to hybrids. The time scales associated with the control rod movement and plasma compression must be quantified, and a clear and practicable action plan should be envisaged. Some of these points can be addressed once a more definitive hybrid system design is conceived.

\section{REFERENCES}

1. Gandini A. HGPT based sensitivity methods for the analysis of subcritical systems. - Ann. Nucl. Energy, 2001, vol. 28 , p. 1193.

2. Gandini A. ADS subcriticality evaluation based on the generalized reactivity concept. - Ann. Nucl. Energy, 2004, vol. 31/7, p. 813.

3. Carta M., Fabrizio V., Falconi L., Gandini A., Iorio M.G., Peluso V., Santoro E., Burgio N. The Power Control Based Subcriticality Monitoring (PCSM) method for ADS reactors. — In: RRFM/IGORR 2016, Berlin, Germany, 13 -17 March 2016. ISBN: 978-92-95064-25-6.

4. Snead L.L., Besmann T.M., Collins E.D., Bell G. Deep Burn Development of Transuranic Fuel for High-Temperature HeliumCooled Reactors. July 2010; DOI 10.2172/1023825.

5. Sakharov A. Memoirs. - 1978, translated by R. Lourie (Knopf, 1990).

6. Bethe H. - Physics Today, 1979, vol. 32, p. 44-51.

7. Bethe H. The Fusion Hybrid Reactor. Report SAND 81-1265, August 1981 (Colloquium at Sandia National Laboratories, United States Department of Energy, September 19, 1980).

8. Proceedings of FUNFI3 - 3rd International Conference on Fusion-Fission Subcritical Systems for Waste Management and Safety. Hefei, Anhui, China, 19-21 November 2018, Edited by A. Pizzuto and F.P. Orsitto, ENEA, ISBN: 978-88-8286-384-5 (2019).

9. Rimpault G., Plisson D., Tommasi J., Jacqmin R., Rieunier J.-M., Verrier D., Biron D. The ERANOS code and data system for fast reactor neutronic analyses. - In: International Conference PHYSOR 2002. Seoul, Korea.

10. Di Palo L. RC-1 Reattore 1 MW - progetto definitivo e rapporto di sicurezza. CNEN Centro Studi Nucleari Casaccia, 1966 (in Italian).

11. Gho C.J., Palmiotti G. BISTRO: Bidimensionnel SN transport optimisé. Un programme bidimensionnel de transport SN aux différences finies, Note No 1: Définition des algorithmes pour la géométrie X-Y, Note Technique, CEA, SPRC/LEPh 84-270, 1984 (in French).

12. Usachev L.N. Equations for the neutron value, reactor kinetics and perturbation theory. — In: Proceedings of International Conference on Peaceful Uses of Atomic Energy, UNO, Geneva, Switzerland, 8-20 August 1955, vol. 5, p. 503. (Reports of soviet delegation on International Conference on Peaceful Uses of Atomic Energy. - M.: Pub.house of the USSR AS, 1955.308 p. (in Russian).

13. Carta M., D’Angelo A. - Nucl. Sci. Eng., 1999, vol. 133, p. 3.

14. Koning A., Forrest R., Kellett M., Mills R., Henriksson H., Rugama Y. The JEFF-3.1 Nuclear Data Library. — OECD/NEA, 2006, № 6190 .

15. Freidberg J.P. Plasma Physics and Fusion Energy. — Cambridge University Press, 2007.

16. Greene J.M., Johnson J.L., Weimer K.E. Tokamak equilibrium. — Phys. Fluids, 1971, vol. 14, № 3, p. 671 —683.

17. Tait G., Bell M.G., Bell J. Adiabatic Toroidal Compression and Free-Expansion Experiments in TFTR. — International Atomic Energy Agency, 1985.

18. Golant V.E. The investigation of adiabatic compression in tokamaks. - Plasma Phys. Control. Fusion, 1984, vol. 26, p. 77-86.

19. Furth H.P., Yoshikawa S. Adiabatic compression of tokamak discharges. — Phys. Fluids, 1970, vol. 13, № 10, p. 2593 - 2596.

20. Li Ge.The inductance of compressed plasma. - Nucl. Fusion, 2015, vol. 55, p. 033009.

21. Freidberg J.P. Ideal Magnetohydrodynamics. — Plenum, 1987.

\section{AUTHORS}

N. Burgio, First Researcher, Master of Art Degree (Chemistry) and PhD student (Energy and Environment), ENEA - Italian National Agency for New Technologies, Energy and Sustainable Economic Development - C.R. Casaccia, via Anguillarese 301, 00123 S. Maria di Galeria, Rome, Italy, and Sapienza University of Rome, Department of Astronautical, Electrical and Energy Engineering, Corso Vittorio Emanuele II 244, Rome, Italy, nunzio.burgio@enea.it

Mario Carta, Research Manager, PhD (Physics), ENEA — Italian National Agency for New Technologies, Energy and Sustainable Economic Development - C.R. Casaccia, via Anguillarese 301, 00123 S. Maria di Galeria, Rome, Italy, mario.carta@enea.it

Valentina Fabrizio, Researcher, PhD (Energy and Environment), ENEA — Italian National Agency for New Technologies, Energy and Sustainable Economic Development — C.R. Casaccia, via Anguillarese 301, 00123 S. Maria di Galeria, Rome, Italy, valentina.fabrizi@enea.it 
Luca Falconi, Researcher, Master of Art (Physics), ENEA - Italian National Agency for New Technologies, Energy and Sustainable Economic Development — C.R. Casaccia, via Anguillarese 301, 00123 S. Maria di Galeria, Rome, Italy, luca.falconi.1@enea.it

Augusto Gandini, Honorary Fellow, PhD (Physics), Sapienza University of Rome, Department of Astronautical, Electrical and Energy Engineering, Corso Vittorio Emanuele II 244, Rome, Italy, augusto.gandini@uniroma1.it

Renato Gatto, Associate Professor, Master of Art (Physics) and PhD (Nuclear Engineering), Sapienza University of Rome, Department of Astronautical, Electrical and Energy Engineering, Corso Vittorio Emanuele II 244, Rome, Italy, renato.gatto@ uniroma1.it

Vincenzo Peluso, Researcher, PhD (Energy and Environment), ENEA — Italian National Agency for New Technologies, Energy and Sustainable Economic Development — C.R. Bologna, Via dei Martiri di Monte Sole, 4 - 40129, Bologna, Italy, vincenzogiuseppe.peluso@enea.it

Emilio Santoro, Consultant, Master (Nuclear Physics), ENEA — Italian National Agency for New Technologies, Energy and Sustainable Economic Development — C.R. Bologna, Via dei Martiri di Monte Sole, 4 - 40129, Bologna, Italy, emilio.santoro1618@gmail.com

Maria Beatrice Sciarretta, Business developer, Master of Art (Energy Engineering), ENEL, Viale Regina Margherita 125, Rome, Italy, mbsciarretta@gmail.com 